\title{
barrage de Diama \\ les remblais d'essais
}

\author{
par \\ J. Costaz \\ Coyne \& Bellier
}

\section{Le site et le projet}

Dans le cadre du projet d'aménagement intégré, lancé par l'Organisation pour la Mise en Valeur du fleuve Sénégal, le barrage mobile de Diama, se situe dans le delta du fleuve près de Saint-Louis du Sénégal.

Le projet comporte un barrage mobile, construit à terre, dans la concavité d'un méandre rive gauche, des endiguements latéraux amont sur les berges du fleuve et une route d'accès et de liaison reliant Saint-Louis à Nouakchott (voir plan de situation de la figure 0 ). Ce projet est en cours d'études par le groupement d'Ingénieurs Conseils, SOGREAH - Coyne et Bellier - BCEOM. Le Groupement d'études s'est adjoint les services du CEBTP-Paris, en ce qui concerne les essais de laboratoire. L'OMVS s'est, de plus, assuré les services de $M$. L'Hériteau, du bureau Mécasol, à titre d'expert sur les problèmes de géotechnique.

Au droit du barrage mobile, les remblais d'accès, formant digues de fermeture de la retenue, se développent sur $0,60 \mathrm{~km}$ en rive gauche et sur $1,50 \mathrm{~km}$ en rive droite. Sur le site, les cotes du terrain naturel, se situent à $(+1,00)$ environ en dehors des points bas (aux passages des marigots) qui se situent entre $(0,00)$ et $(+0,50)$. Les remblais d'accès à construire doivent ménager une plateforme de $20 \mathrm{~m}$ de largeur, à la cote $(+4,00)$ du projet routier final. II s'agit donc de construire des digues de $3,50 \mathrm{~m}$ à $4,00 \mathrm{~m}$ de hauteur totale, hors provisions pour tassement.

\section{Les fondations}

\subsection{Géométrie}

Les remplissages fluvio-deltaïques rencontrés sur l'axe des digues, d'après les reconnaissances effectuées en rive gauche, dans la concavité du méandre, sont formés de : a) matériaux vasards très compressibles entre la cote du TN et la cote $(-10,00)$ environ, sous une croûte de dessication superficielle d'une épaisseur de l'ordre de $0,50 \mathrm{~m}$ à $0,70 \mathrm{~m}$,

b) sables plus ou moins compacts entre les cotes $(-10,00)$ et $(-14,00)$ environ, c) argiles sableuses fermes entre $(-14,00)$ et $(-16,00)$ à $(-17,00)$

d) un substratum sablo-gréseux sous la cote $(-16,00)$ à $(-17,00)$.

La nappe, compte tenu du fait que le niveau du fleuve, dans le lit mineur, est réglé par la marée, oscille autour de la cote $(0,00)$, entre $(-0,50)$ et $(+0,50)$ environ, en dehors des épisodes de crues de la saison d'hivernage.

\subsection{Caractéristiques d'identification des vases supérieures}

Les analyses granulométriques effectuées (voir figure $n^{\circ} 1$ ) montrent des matériaux assez hétérogènes s'étalant entre des vases franches et des sables très fins (d'origine éolienne probablement, du type dunaire), en passant par des limons plus ou moins sableux. II n'a pas été possible de corréler d'un sondage à l'autre les couches à tendance plus sableuse, ce qui reflète les variations de facies latéraux propres à ce genre de dépôts littoraux, dans lesquels des "lentilles » sont plus courantes que des couches continues traduisant une stratification horizontale.

Les caractéristiques de plasticité des matériaux à tendance vasarde donnent des indices de plasticité variant entre $40 \%$ et $80 \%$ autour d'une moyenne IP $=60 \%$ (écart-type $=11 \%$ sur vingt trois mesures), pour des limites de liquidité variant entre 60 et $110 \%$ autour d'une moyenne $W_{L}=90 \%$ (écart-type $=15 \%$ ). Les points représentatifs se placent au-dessus de la ligne $A$ de Casagrande (voir figure $n^{\circ} 2$ ).

Les poids volumiques secs sont assez variables entre $7 \mathrm{kN} / \mathrm{m}^{3}$ pour les vases pures et $13 \mathrm{kN} / \mathrm{m}^{3}$ pour les sables (voir figure $n^{\circ} 3$ ). Les teneurs en matières organiques sont de l'ordre de $3 \%$ à $5 \%$, (deux mesures).

\section{3 État en place des vases supérieures}

Les teneurs en eau, in situ, sont très proches des limites de liquidité correspondantes (voir figure $n^{\circ} 3$ ), l'indice de consistance varie entre 0 et 0,50 a 0,60 au maximum.

La cohésion non drainée $\left(C_{u}\right)$, obtenue à l'aide d'essais scissométriques effectués en rive gauche tous les mètres sur vingt trois forages de dix mètres de profondeur, est variable suivant la profondeur autour de la courbe moyenne, définie par $\bar{C}_{u}$ (moyenne des mesures au niveau 


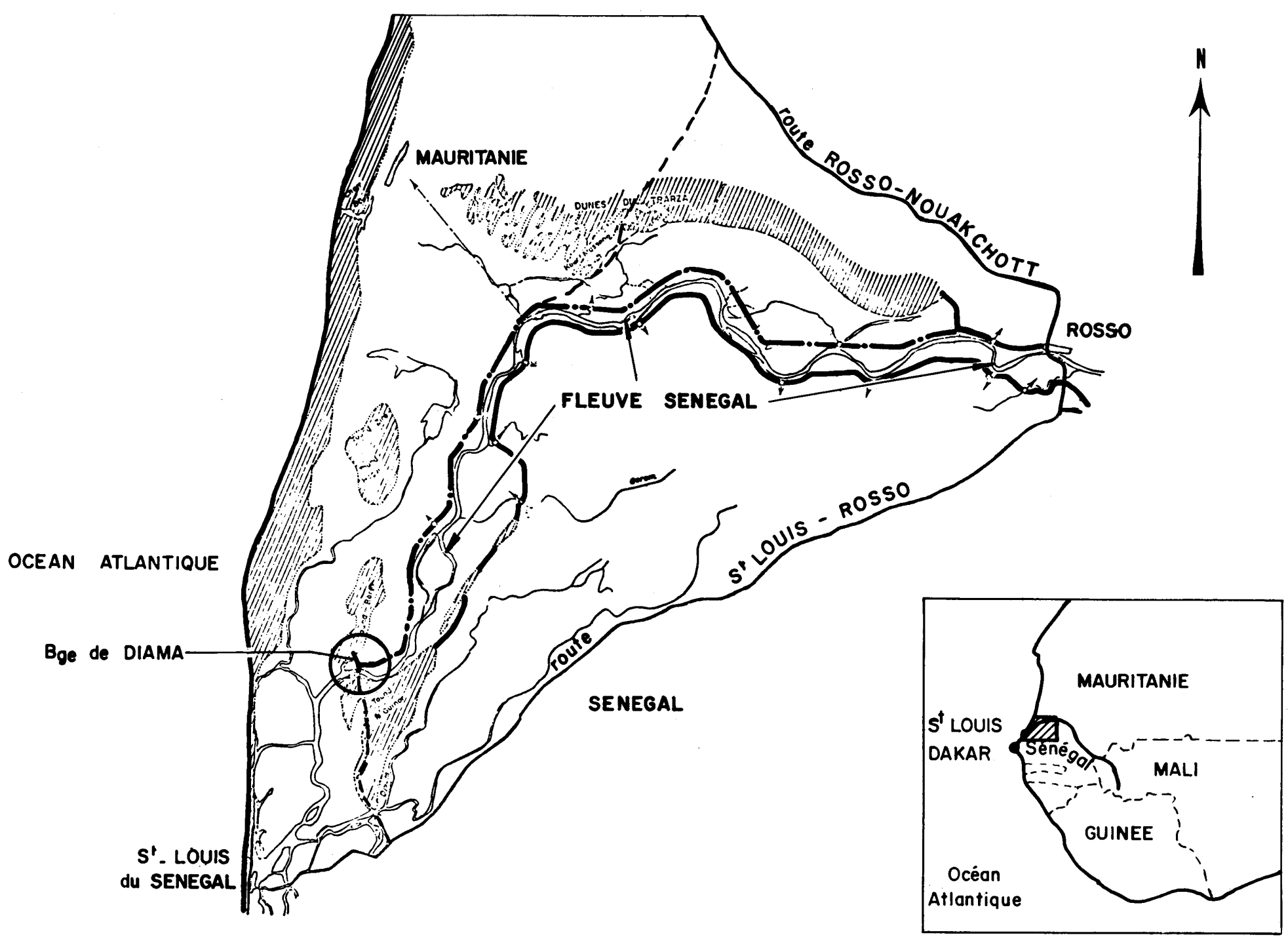

Fig. 1 Granulométrie des matériaux du site

1 : Sables fins ; 2 : Argiles sableuses ; 3 : Vases

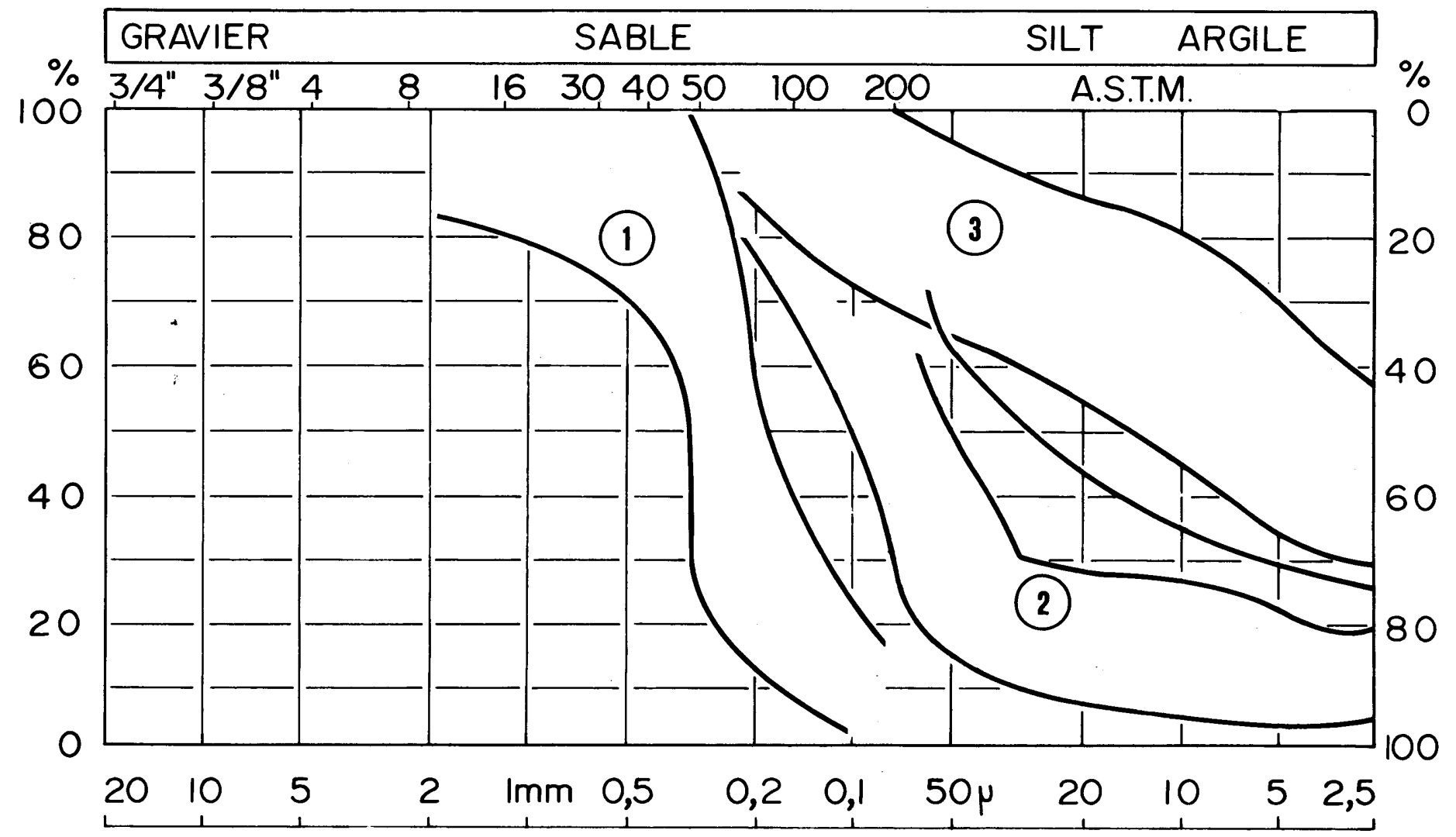




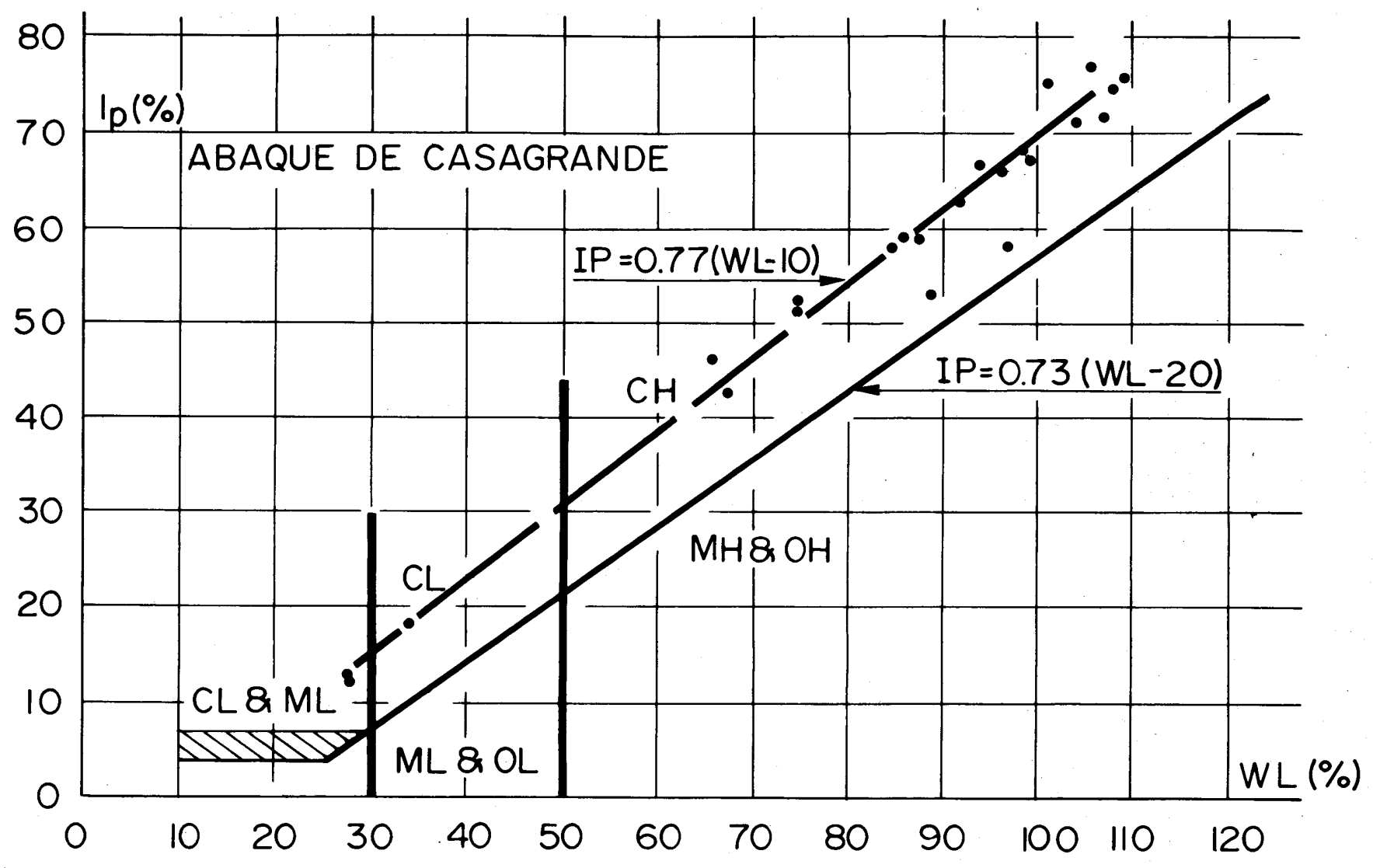

Fig. 2 Plasticité des matériaux du site

Fig. 3 Etat en place des vases
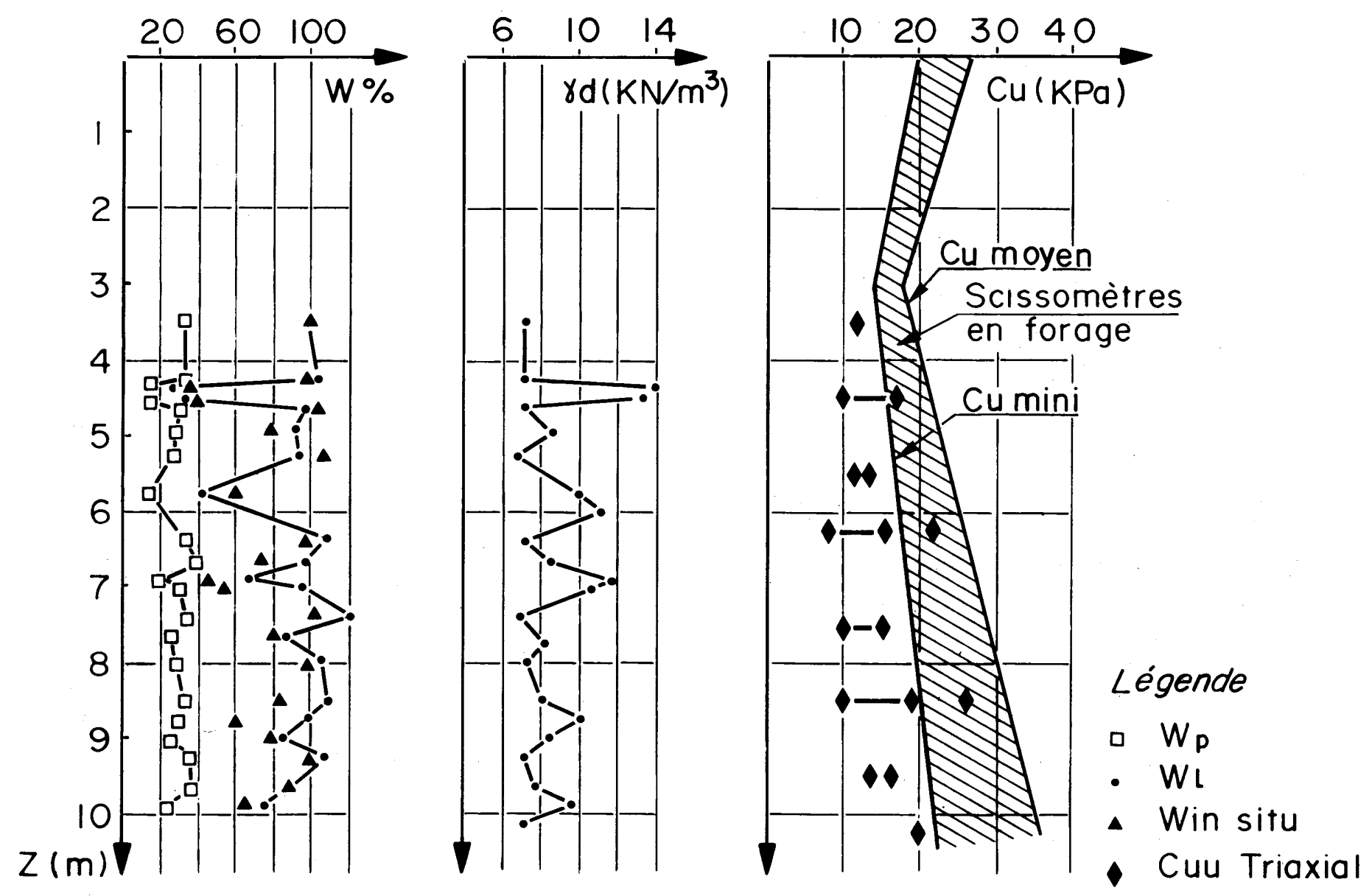
considéré). La courbe "minimum», définie par $\bar{C}_{u}-s$ (moyenne moins écart type au niveau considéré) a pu ainsi être définie :

$\begin{array}{ll}\text { cote du TN } & \mathrm{Cu}=20 \mathrm{KPa} \\ \text { profondeur } 3 \mathrm{~m} & \mathrm{Cu}=14 \mathrm{KPa} \\ \text { profondeur } 10 \mathrm{~m} & \mathrm{Cu}=22 \mathrm{KPa}\end{array}$

Cette courbe «minimale » est toujours supérieure à la droite $C_{u \text { min }}=0,32 \sigma_{\text {vo }}^{\prime}$ (obtenue en supposant $\gamma^{\prime}=6 \mathrm{KN} / \mathrm{m}^{3}$ sur toute la hauteur des fondations, en joignant l'origine avec le point Cumini à $10 \mathrm{~m}$ de profondeur). Cette droite correspondrait, (encore que l'absence de résultats de densité dans les trois mètres superficiels, ne nous permette pas de tracer avec précision un diagramme complet de la contrainte effective en fonction de la profondeur), à une valeur de la résistance au

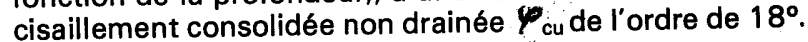

Le fuseau ainsi défini, à partir des essais en forage, est donné dans la figure 3 , qui montre que le rapport entre $C_{u}$ minimale (définie par $\mathrm{C}_{u}-\mathrm{s}$ ) et $\overline{\mathrm{C}}_{\mathrm{u}}$ est de l'ordre de 0,70 à 0,80 suivant la profondeur.

\subsection{Caractéristiques mécaniques à court terme obtenues en laboratoire}

Les essais triaxiaux non consolidés non drainés (de type UU) ont donné des résultats presque systématiquement inférieurs à ceux des scissomètres en forage.

Les résultats des essais de laboratoire sont systématiquement supérieurs à une droite $C_{u}=0,210 \sigma^{\prime}$ vo, qui correspondrait à une valeur $\varphi_{\mathrm{cu}}=12^{\circ}$ environ.

Une étonnante homogénéité des résultats autour d'une valeur de $\varphi_{\mathrm{cu}}=10^{\circ}$ (sur douze valeurs mesurées), a été trouvée à partir des essais triaxiaux consolidés non drainés. La valeur statistique de la résistance non drainée déduite des essais triaxiaux et celle mesurée au scissomètre sont différentes mais compte tenu du nombre réduit des essais de laboratoire par rapport au nombre des essais scissométriques, il est illusoire d'essayer de définir un coefficient de corrélation entre les deux familles de résultats obtenus.

\subsection{Caractéristiques œdométriques}

Les essais œdométriques, effectués en laboratoire, ont donné les résultats de la figure 4. Notons qu'en raison de la très grande sensibilité des matériaux (de l'ordre de 4 à 6), les échantillons "intacts» des prélèvements dans les matériaux les plus plastiques ont été le siège d'un remaniement, dans cinquante pour cent des cas (les essais ont eu lieu sur des échantillons ayant subi un transfert Dakar-Paris).

Les valeurs des pressions de préconsolidation sont difficilement interprétables dans le cas présent et ne sont pas toujours représentatives d'un matériau normalement consolidé, ce qui est très vraisemblablement le cas.

\section{Stabilité à court terme du projet - Próvision}

\subsection{Rupture par poinçonnement généralisé}

Compte tenu de la géométrie du projet, définie antérieurement, le schéma d'écoulement du type Prandtl n'est pas valable. En supposant que l'interface entre le plancher des vases et les sables inférieurs est rugueux, dans les conditions du modele de Mandel et Salençon, [1] les calculs de la force portante des vases ont été effectués par application de la formule classique :

$$
q_{\mathrm{adm}}=\mathrm{Fc} \mathrm{Nc}^{\mathrm{C}_{\mathrm{u}}}
$$

où $\mathrm{Nc}=(\pi+2)$ d'après Terzaghi, $\mathrm{Fc}=$ coefficient correcteur de Mandel et Salençon (fonction de la largeur du remblai et de l'épaisseur des vases) et $F=$ facteur de sécurité sur la portance.

Le problème principal reste dans le choix de la valeur de $\mathrm{Cu}$ à retenir pour les calculs. En tenant compte de la correction, empirique à apporter aux valeurs de $\mathrm{Cu}$, déduites des essais au scissometre en forage, on obtient :

$$
\mathrm{Cu}_{\text {calcul }}=\mu \mathrm{Cu}_{\text {scisso }}
$$

où le terme correcteur $\mu$ est celui recommandé par L. Bjer-

Fig. 4 Caractéristiques œdométriques

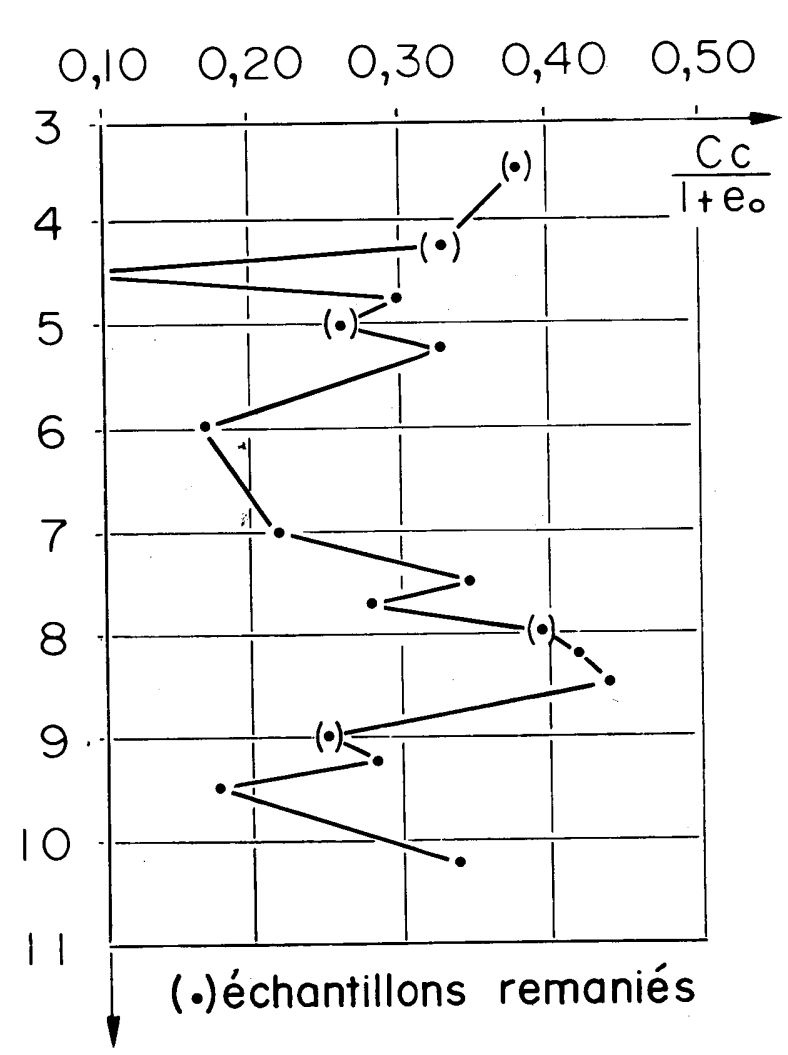

$Z(\mathrm{~m})$

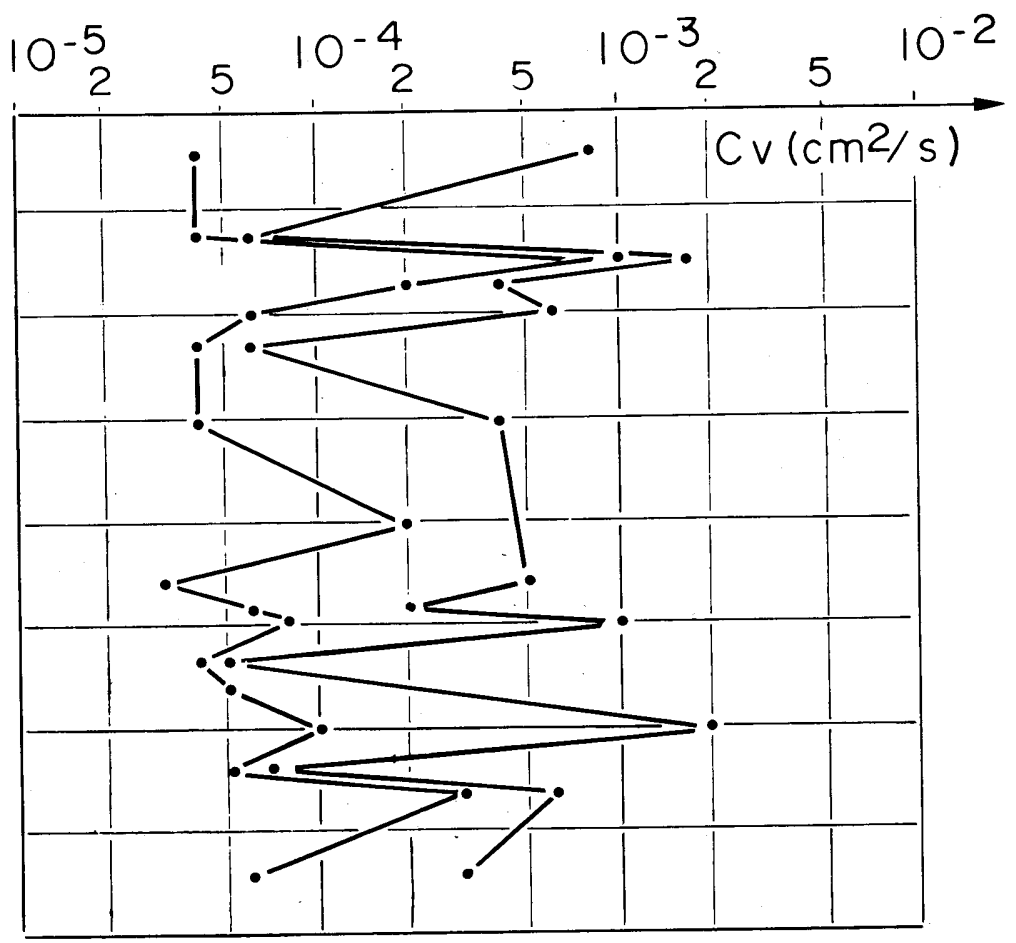

Fourchette de $C_{v}$ pour $\sigma_{c}^{\prime}=80 \mathrm{KPa}-160 \mathrm{KPa}$ $\mathrm{Z}(\mathrm{m})$ 
rum [2] qui varie entre 0,70 et 0,80 pour un indice de plasticité variant entre 50 et $70 \%$.

Dans ces conditions, pour un facteur de sécurité de l'ordre de $F=1,30$, il est possible d'associer un doublet $(\mu, h r)$ dont les valeurs calculées (voir figure 5 ), en supposant $\mathrm{Cu}_{\text {sciseo }}=18 \mathrm{KPa}$ isotrope sur toute l'épaisseur des vases supérieures, sont les suivantes :

$$
\begin{array}{lll}
\mathrm{F}=1,30 & \mathrm{hr}=3,00 \mathrm{~m} & \mu=0,70 \\
& \mathrm{hr}=3,50 \mathrm{~m} & \mu=0,80 \\
& \mathrm{hr}=4,50 \mathrm{~m} & \mu=1,00
\end{array}
$$

où $\mathrm{hr}=$ hauteur du remblai, $\gamma \mathrm{r}=18 \mathrm{KN} / \mathrm{m}^{3}$

L'effet de la croute de dessication de surface ne peut être pris en compte au niveau de ces calculs de portance. Cette croate est d'ailleurs généralement fissurée sur 0,15 à $0,30 \mathrm{~m}$, au moins.

\subsection{Rupture rotationnelle locale}

A l'aide d'un calcul en contraintes totales par la méthode de Fellenius, où la cohésion non drainée varie avec la profondeur suivant les courbes moyennes et minimales (définies dans la figure $n^{\circ} 3$ ), pour un même facteur de sécurité $F=1,30$, les valeurs calculées (voir figure 5 ) sont les suivantes:

$F=1,30$

$\begin{array}{ll}\mathrm{C}_{\mathrm{u}}=\mathrm{C}_{\mathrm{u}} \text { moyen } & \mathrm{hr}=5,25 \mathrm{~m} \\ \mathrm{C}_{\mathrm{u}}=\mathrm{C}_{\mathrm{u}}-\mathrm{s}=\mathrm{C}_{\mathrm{u} \min } & \mathrm{hr}=3,75 \mathrm{~m}\end{array}$

L'effet de la dessication dans la partie supérieure est pris en compte, dans la mesure où l'on a traduit la courbe $(\mathrm{Cu}, \mathrm{z})$ dans les calculs, ce calcul néglige toutefois la couche mince superficielle $(0,50$ a $0,70 \mathrm{~m})$, fortement surconsolidée par dessication mais généralement fissurée en surface.

\section{Tassements des vases sous charge}

\subsection{Modèle retenu}

Le modèle d'une couche unique avec drainage par les deux faces, du type de celui de Terzaghi, a été retenu, au niveau de la prévision à l'avant projet sommaire.

La face supérieure, au niveau du terrain naturel, est en fait couverte pour la croate de dessication de surface. L'état de fissuration de surface permet de supposer qu'il peut y avoir drainage superficiel.

L'interface inférieure entre les vases et les sables, vers le niveau $(-10,00)$, présente un contraste de perméabilités justifiant l'hypothèse du drainage inférieur. La perméabilité " en grand " des sables, mesurée par un essai de pompage, correspondant donc à une perméabilité horizontale, est de l'ordre de KS = $2 \times 10^{-4} \mathrm{~m} / \mathrm{s}$.

Dans l'épaisseur de la couche des vases, dans la mesure où les "lentilles" sableuses peuvent, en quelque sorte, se relayer les unes les autres, il est possible que des lits drainants existent dont le modele isotrope ne peut rendre compte.

\subsection{Caractéristiques œdométriques retenues}

Comme le montre la figure 4, il est bien difficile, pour ne pas dire impossible, de considérer le milieu comme homogène sur toute son épaisseur compte tenu de la dispersion des résultats obtenus, qui ne fait que traduire l'hétérogénéité des matériaux (et des prélèvements plus ou moins remaniés). Les caractéristiques de compressibilité " moyennes » pourraient être, pour le coefficient de compressibilité,

$$
\frac{C c}{1+e_{0}}=0,30 \pm 0,08
$$

Fig. 5 Projet - Comparaison entre poinçonnement généralisé et rupture rotationnelle

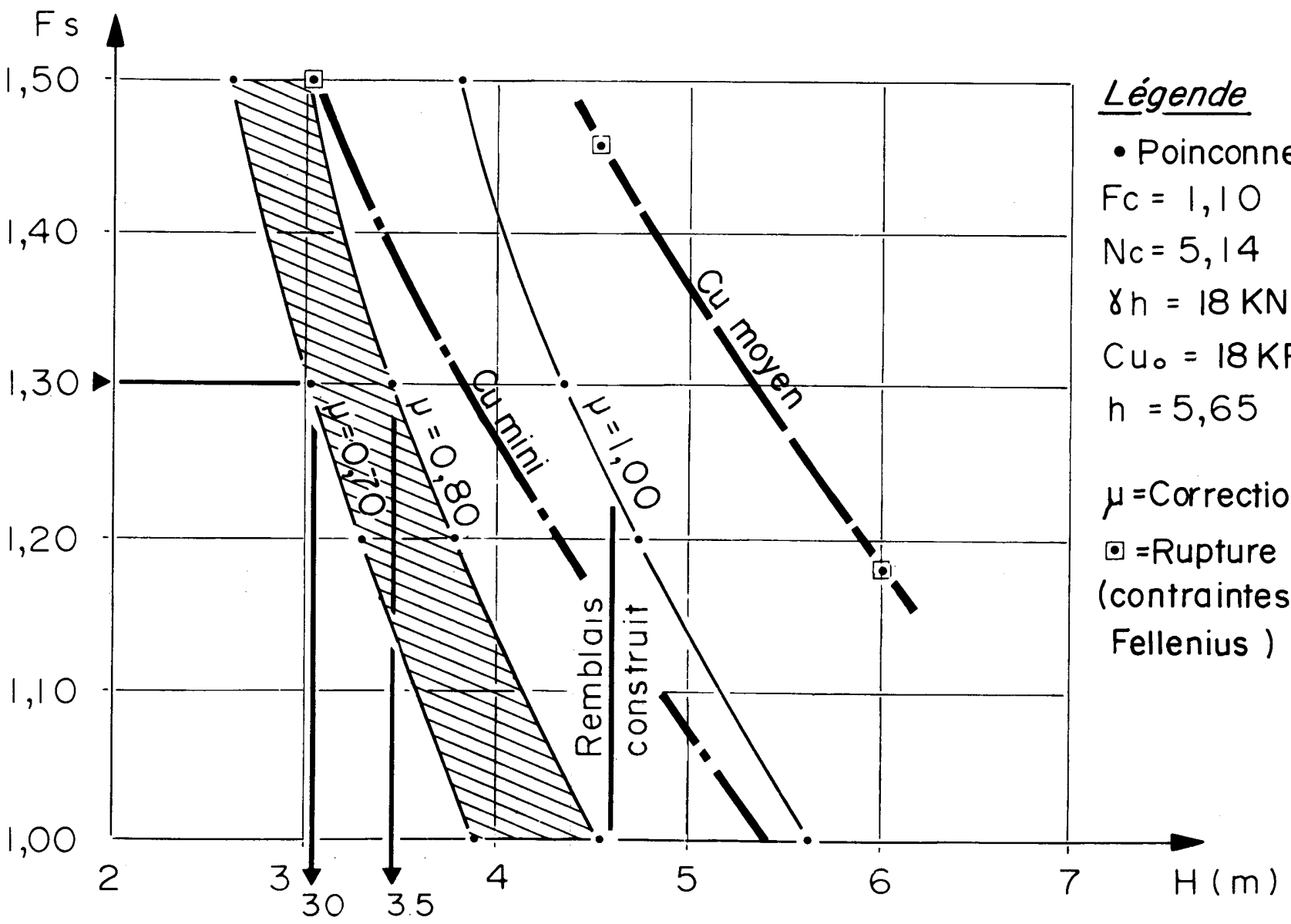


quant à la valeur du coefficient de consolidation, il peut être défini par les valeurs extrêmes suivantes:

$$
5 \times 10^{-5} \mathrm{~cm}^{2} / \mathrm{s}<\mathrm{C}_{\mathrm{v}}<5 \times 10^{-4} \mathrm{~cm}^{2} / \mathrm{s}
$$

Aucun essai de consolidation radiale n'a été effectué.

\subsection{Prévision au stade préliminaire}

Avec les paramètres de calcul ainsi retenus, appliqués au modèle simplifié, les résultats prévisionnels pour un remblai de $3,50 \mathrm{~m}$ de hauteur moyenne, (soit une surcharge de $63 \mathrm{KPa}$ ), ont été les suivants:

a) tassement ultime à prévoir de l'ordre de $1,50 \mathrm{~m}$ à $1,60 \mathrm{~m}$ en supposant le matériau isotrope normalement consolidé,

b) pourcentage du tassement total obtenu au bout de 4 ans (délai global du chantier du barrage de Diama), variant entre $17 \%$ à $55 \%$ suivant le coefficient de consolidation admis.

\section{Conclusions préliminaires}

\subsection{Impossibilité de construire le remblai en une seule phase}

L'ordre de grandeur de tassements à prévoir montre donc que la hauteur initiale à donner aux digues à construire serait de l'ordre de $5,50 \mathrm{~m}$ a $6,00 \mathrm{~m}$, en incluant une provision pour la totalité du tassement ultime calculé sur le modèle, défini en 4.1. (voir [3] - chapitre 5).

La portance de la fondation, dans ce cas, serait telle que, sans même apporter à l'évaluation de la résistance non drainée la correction de Bjerrum, $(\mu=1,00=$ coefficient correcteur sur la cohésion non drainée, mesurée au scissomètre en forage), le facteur de sécurité serait de l'ordre de $F=0,95$ à 1,05 .
II apparaissait donc impossible de construire en une seule phase les remblais d'accès, avec une sécurité acceptable.

\subsection{Nécessité d'un remblai d'essais}

Compte tenu de ce qui précède, le degré d'incertitude sur le choix des caractéristiques mécaniques est tel que tout raffinement pour analyser par un modèle plus complexe, le comportement d'un matériau apparamment hétérogène, a paru inutile. Un essai en vraie grandeur était donc la seule solution capable d'apporter les éléments de décision nécessaires au niveau du projet détaillé.

Les questions posées sont les suivantes:

a) Est-il possible de construire les remblais d'accès en une seule phase?

b) Quel sera le comportement réel de la fondation, en ce qui concerne les tassements?

Le choix qui reste à faire, au niveau de l'essai en vraie grandeur est donc entre un remblai simple monté jusqu'à rupture, ou un remblai partiel, correspondant à la hauteur strictement nécessaire pour le projet (soit 4,50 m environ) muni d'un dispositif d'auscultation qui reste compatible avec les contraintes de crédit et d'exécution.

Un remblai ausculté, fut proposé et retenu, il fut par la suite décidé de le compléter par un remblai simple monté jusqu'à obtenir la rupture.

\section{Interprétation du remblai à rupture}

Après une reconnaissance scissométrique des fondations le remblai "à rupture " a été monté, sans qu'il n'y ait rupture d'ailleurs, jusqu'à une hauteur de $7,80 \mathrm{~m}$ qui correspond à une surcharge de $133 \mathrm{KPa}$. La plateforme, au niveau du TN a pour dimensions $85 \mathrm{~m} \times 35 \mathrm{~m}$, pour une épaisseur de vases de $10 \mathrm{~m}$ environ. Le matériau de

Fig. 6 Remblais d'essais - Vue en plan

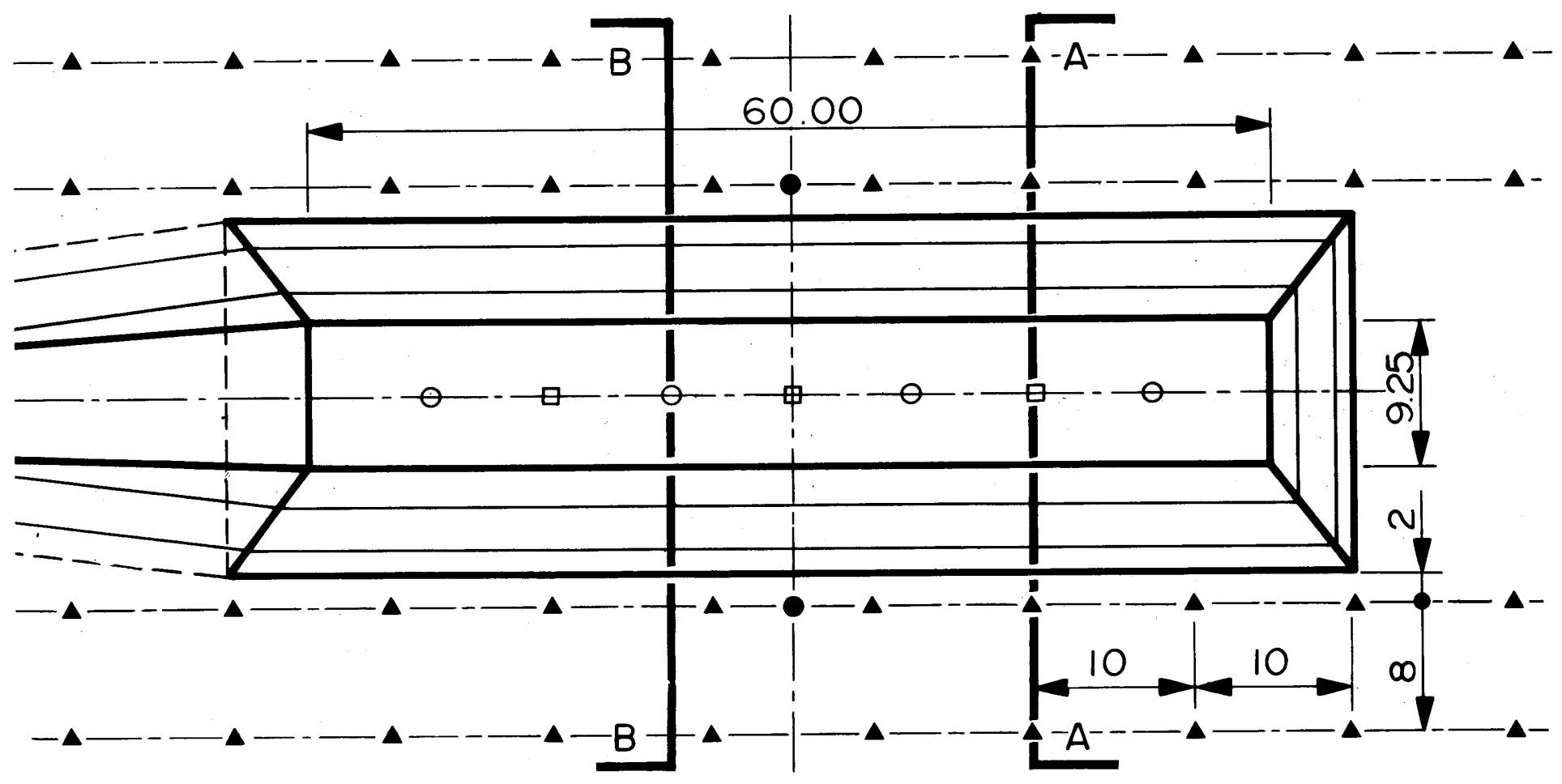


remblai compacté par le passage des engins est un sable silteux de poids volumique $17 \mathrm{KN} / \mathrm{m}^{3}$ et ayant une résistance au cisaillement caractérisé par $\varnothing=35^{\circ}-\mathrm{C}=0$. Les talus latéraux sont de 1,4 H/1V.

Les conditions de stabilité sont, d'après le calcul les suivantes :

a) au poinçonnement généralisé à un doublet $F_{1}=0,85$ avec $\mu_{1}=0,80$ et $F_{2}=1,05$ avec $\mu_{2}=1,00$, pour une cohésion $\mathrm{Cu}_{\text {scisso }}=25 \mathrm{kPa}$ représentative des fondations reconnues.

b) en rupture circulaire, par un calcul en contraintes totales, à un doublet $F_{1}=0,90$ avec une courbe $\mathrm{Cu}$ minimum (définie par $\mathrm{Cu}-\mathrm{s}$ ), et à un doublet $F_{2}=1,10$ avec une courbe $\mathrm{Cu}$ moyenne par la méthode de Fellenius. Dans les mêmes conditions, pour la méthode de Bishop, $F_{1}=1,02$ et $F_{2}=1,22$.

Le rapport entre $C_{u}$ et $C_{u \text { min }}$ varie entre 0,78 et 0,89 suivant le fuseau des résultats scissométriques obtenu dans les forages sous le remblai d'essai. La courbe $\left(C_{u \min }, z\right)$, sous ce remblai d'essais, est définie par la courbe $C_{u}=32 \mathrm{KPa}$ au TN, $C_{u}=20 \mathrm{kPa}$ aे $z=4 \mathrm{~m}$, et $\mathrm{C}_{\mathrm{u}}=35 \mathrm{kPa}$ à $\mathrm{z}=10 \mathrm{~m}$, ce qui la situe légèrement au dessus de la courbe moyenne $\left(C_{u}, z\right)$ définie pour le site et donnée dans la figure 3 .

Notons qu'aucun des alignements topographiques de pied n'a donné de déplacement significatif, soit en planimétrie, soit en nivellement qui puisse traduire une quelconque amorce de mouvement dans la fondation.

\section{Construction du remblai ausculté *}

\subsection{Géométrie}

De façon à éviter les effets de bords [4], pour obtenir des

- Construction assurée par l’Entreprise Sasif Dakar.

Fig. 7

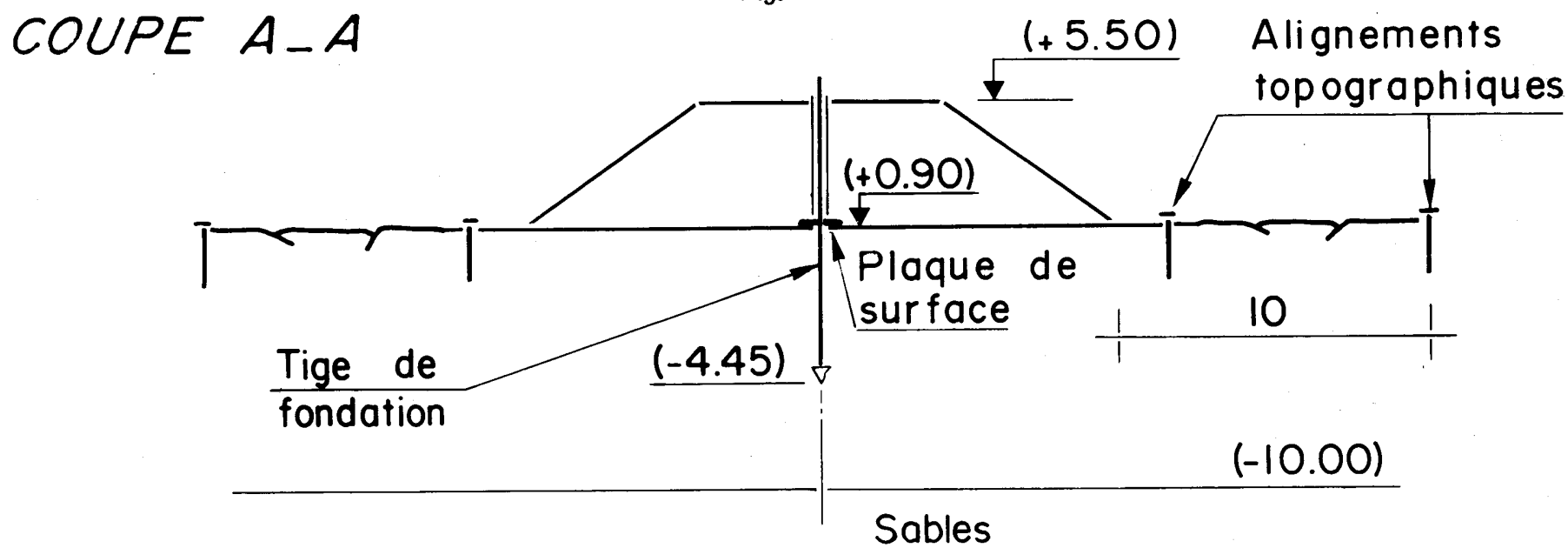

conditions de déformation plane, la géométrie du remblai construit est telle que la plateforme, au niveau du TN a pour dimensions $65 \times 22 \mathrm{~m}$, pour une épaisseur de vases de $9,90 \mathrm{~m}$ (voir figure 6 ).

\subsection{Auscultation}

Pour limiter les contraintes d'exécution de façon à permettre le travail mécanisé nécessaire pour obtenir un chargement rapide; l'auscultation est implantée dans l'axe du remblai, ce qui, d'ailleurs, n'a pas pour effet d'éliminer tout incident sur les tubes de mesures.

L'auscultation comporte, comme le montrent les figures 6 et 7 :

- trois tassomètres simples, fabriqués sur place, destinés à mesurer les tassements à la cote $(+0,90) \mathrm{m}$ au contact remblai-fondation, couplés chacun avec une tige de fondation, destinée à mesurer les tassements au niveau $(-4,45) \mathrm{m}$ au milieu de la couche des vases.

- quatre sondages, ayant servi de reconnaissances scissométriques préalables, équipés chacun de deux l'argile, aux niveaux $(-2,90) \mathrm{m}$ et $(-5,40) \mathrm{m}$.

\subsection{Mesures topographiques}

Les problèmes, posés par les mesures à assurer, ont été essentiellement ceux liés à la topographie, compte tenu de la mouvance généralisée du site qui a rendu la notion de bases «fixes" assez aléatoire. De plus, compte tenu de la hauteur initiale des tubes et tiges de fondation, pendant la première période de construction, donc au cours des mesures initiales, la précision est vraisemblablement assez réduite. cellules de pressions interstitielles (type Glotzl), scellées à

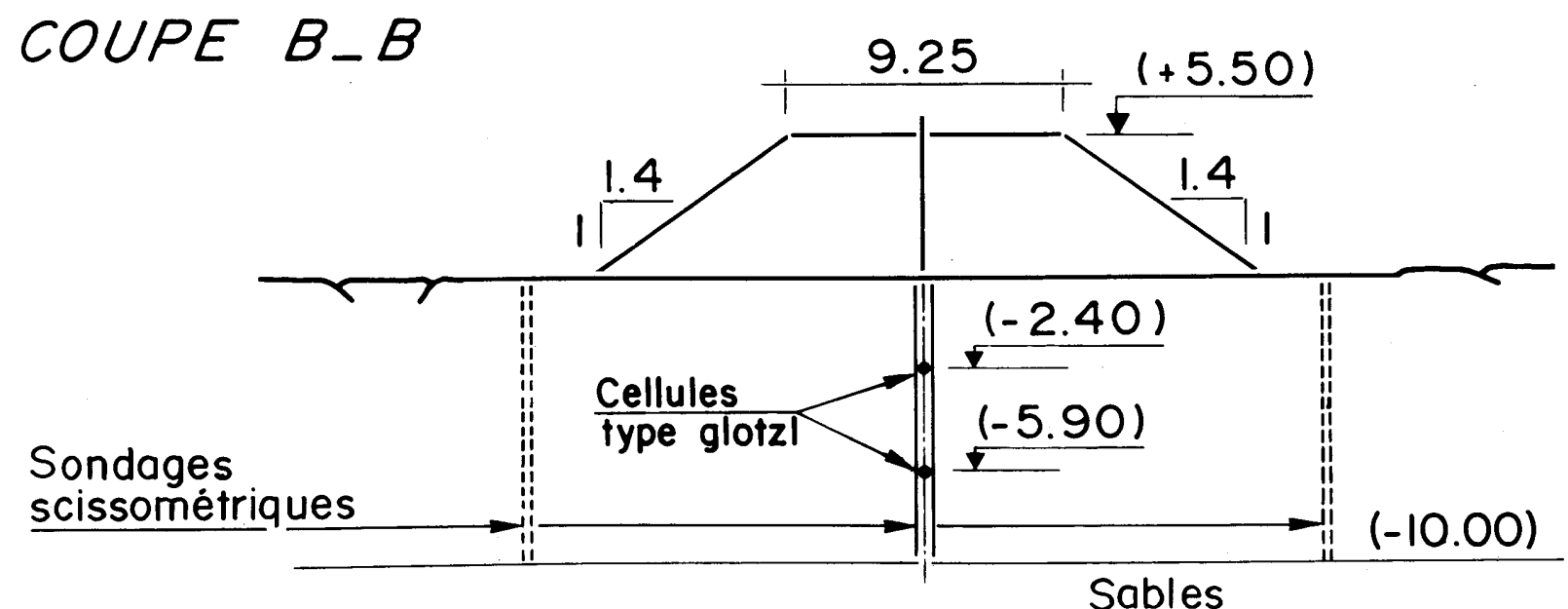


Mesures et essai d'interprétation du comportement du remblai ausculté

\subsection{Tassements mesurés}

Les mesures ont été interrompues au bout de trois mois en raison de la saison des pluies.

Les courbes des tassements mesurés au cours de l'essai sont données dans les figures 8 et 9 qui suivent. Deux phénomènes de type différent ont eu lieu, d'une part avant le septième jour, d'autre part après le septième jour.

Si l'on admet que le processus initial est assimilable à un tassement de type "tassement immédiat", dans la mesure où ce phénomène se produit à volume constant, des déformations immédiates en pied du remblai auraient dues être perçues ce qui n'a pas été le cas. La croûte superficielle a-t-elle, tout simplement, masqué les signes de ces déformations, en provoquant un "étalement » des déformées?

La deuxième phase du développement des tassements est vraisemblablement du type " tassement de consolidation », dont les mesures sont données par la figure 9. Les résultats sont assez bien encadrés par les valeurs, calculées sur un modèle simplifié du type Terzaghi, en supposant un drainage par les deux faces, correspondant à $\frac{\mathrm{C}_{0}}{1+\mathrm{e}_{\mathrm{o}}}=0,20$ à 0,30 suivant la cote avec des valeurs de $\mathrm{C} v$ de l'ordre de $5 \times 10^{-4}$ aे $8 \times 10^{-4} \mathrm{~cm}^{2} / \mathrm{s}$. Inversement, en supposant, d'après les essais de laboratoire que le comportement " moyen " on grand des vases peut être représenté par un milieu homogène où $\mathrm{Cv}=10-4 \mathrm{~cm}^{2} / \mathrm{s}$, il n'est plus possible d'expliquer les mesures par le seul tassement "primaire" qui ne couvre plus qu'environ $40 \%$ des valeurs mesurées sous le remblai. Le comportement du terrain correspond donc à la valeur la plus forte de la fourchette du $\mathrm{C}_{v}$ donnée par le laboratoire.

\subsection{Pressions interstitielles}

Les surpressions interstitielles mesurées sont données par la figure 10, sous forme des fuseaux définis aux niveaux auscultés. Surpression s'entend au sens de pression au-dessus de la pression neutre définie-par l'existence d'une nappe sous la cote $(0,00)$.

Les valeurs mesurées en fin de construction n'atteignant qu'environ $40 \%$ à $60 \%$ de la surcharge, créée par le remblai, les vitesses de dissipation des pressions interstitielles ne correspondent pas du tout avec les valeurs calculées suivant la théorie simplifiée. La vitesse de dissipation est beaucoup plus importante que ne le laisse prévoir les essais de laboratoire, de plus la dissipation en cours de construction, a un effet "réducteur" sensible sur la pression maximum atteinte en fin de chargement.

L'étendue des fuseaux reflète l'hétérogénéité des conditions de drainage dans la masse du remplissage vasard. En particulier, il est vraisemblable qu'un drainage latéral, se superposant au drainage vertical supposé par la théorie simplifiée, a eu lieu, conduisant à une redistribution de la pression interstitielle après la fin de la construction [6]

Fig. 8 Terrassements mesurés

$H(m)$

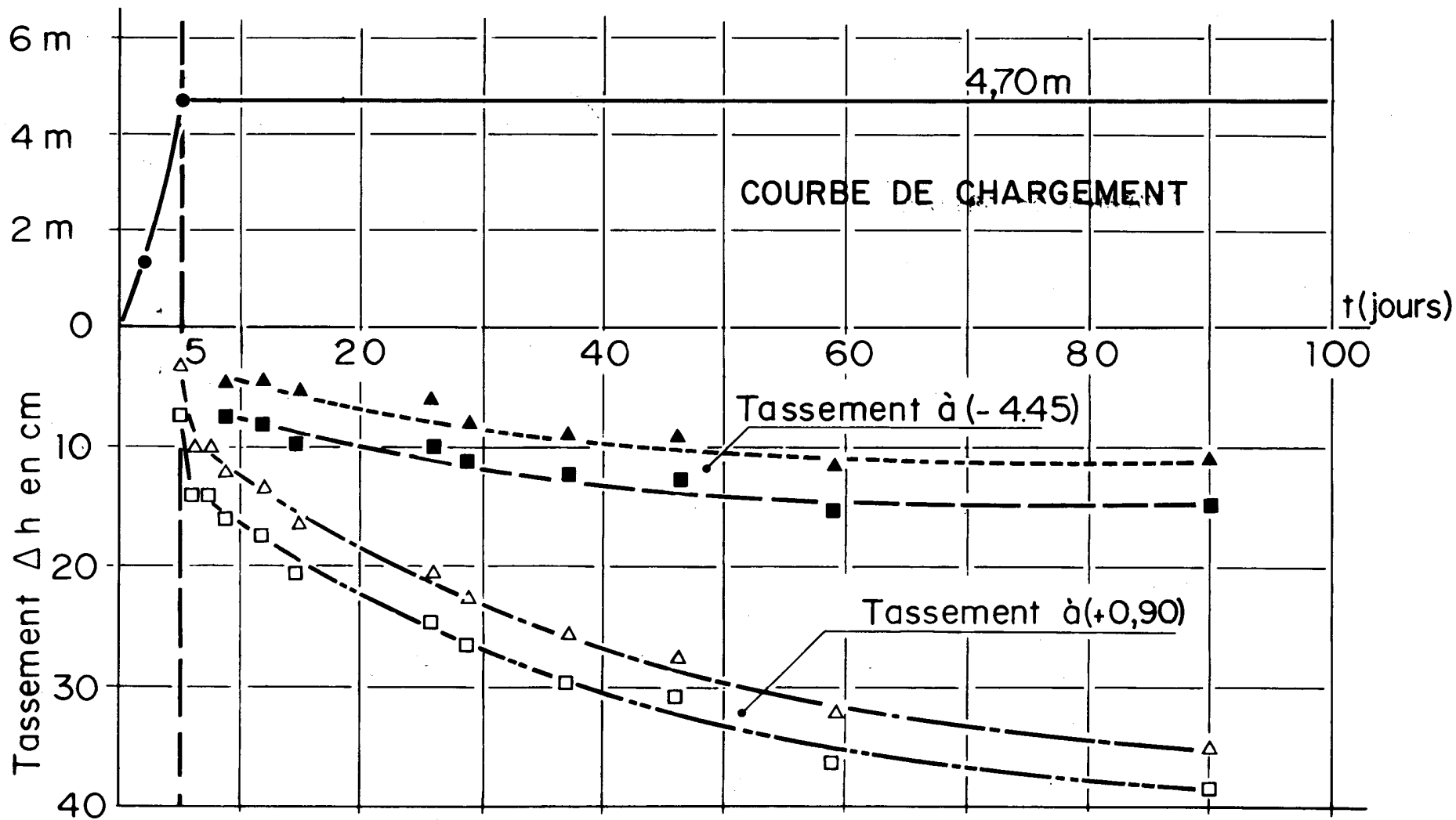


MESURES DE TASSEMENT RESULTATS BRUTS

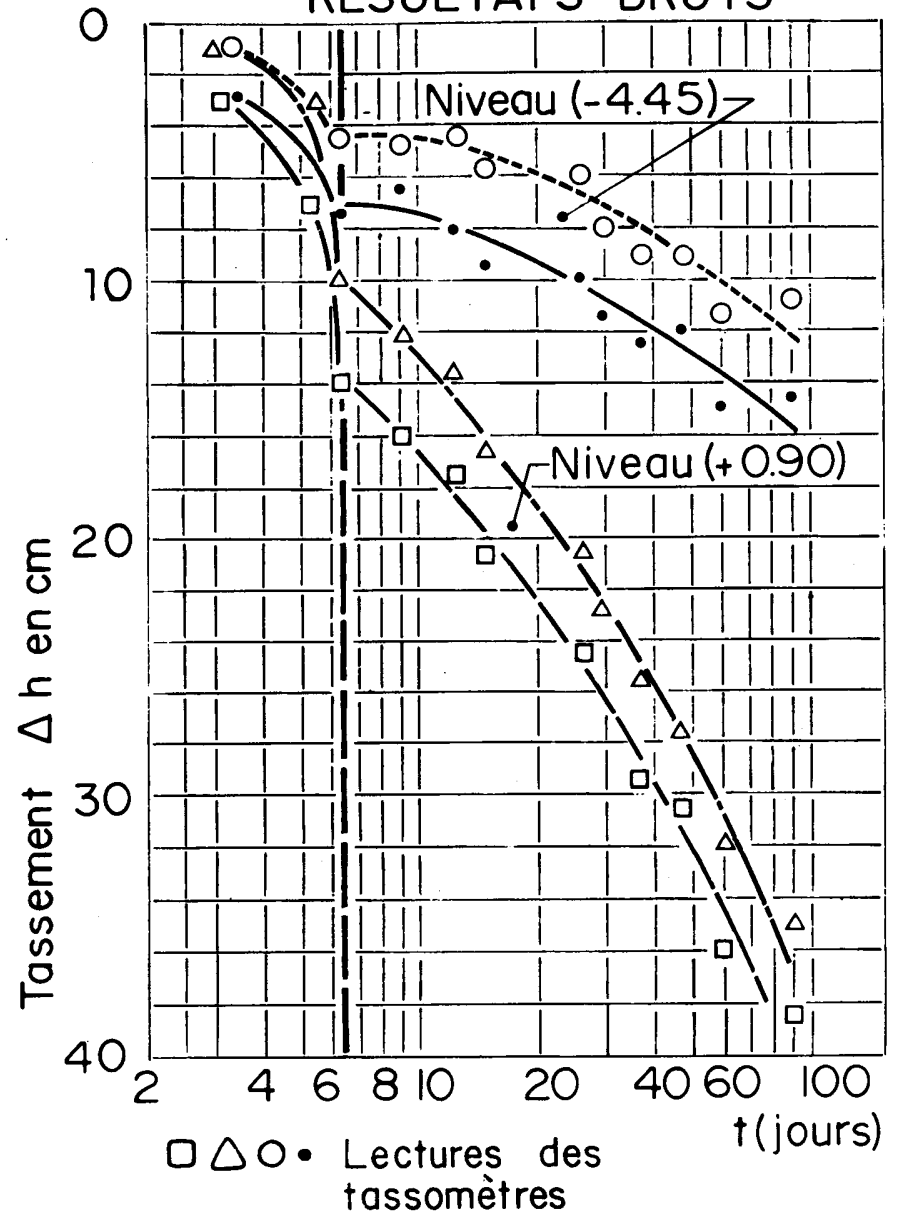

\section{TASSEMENT DE CONSOLIDATION}

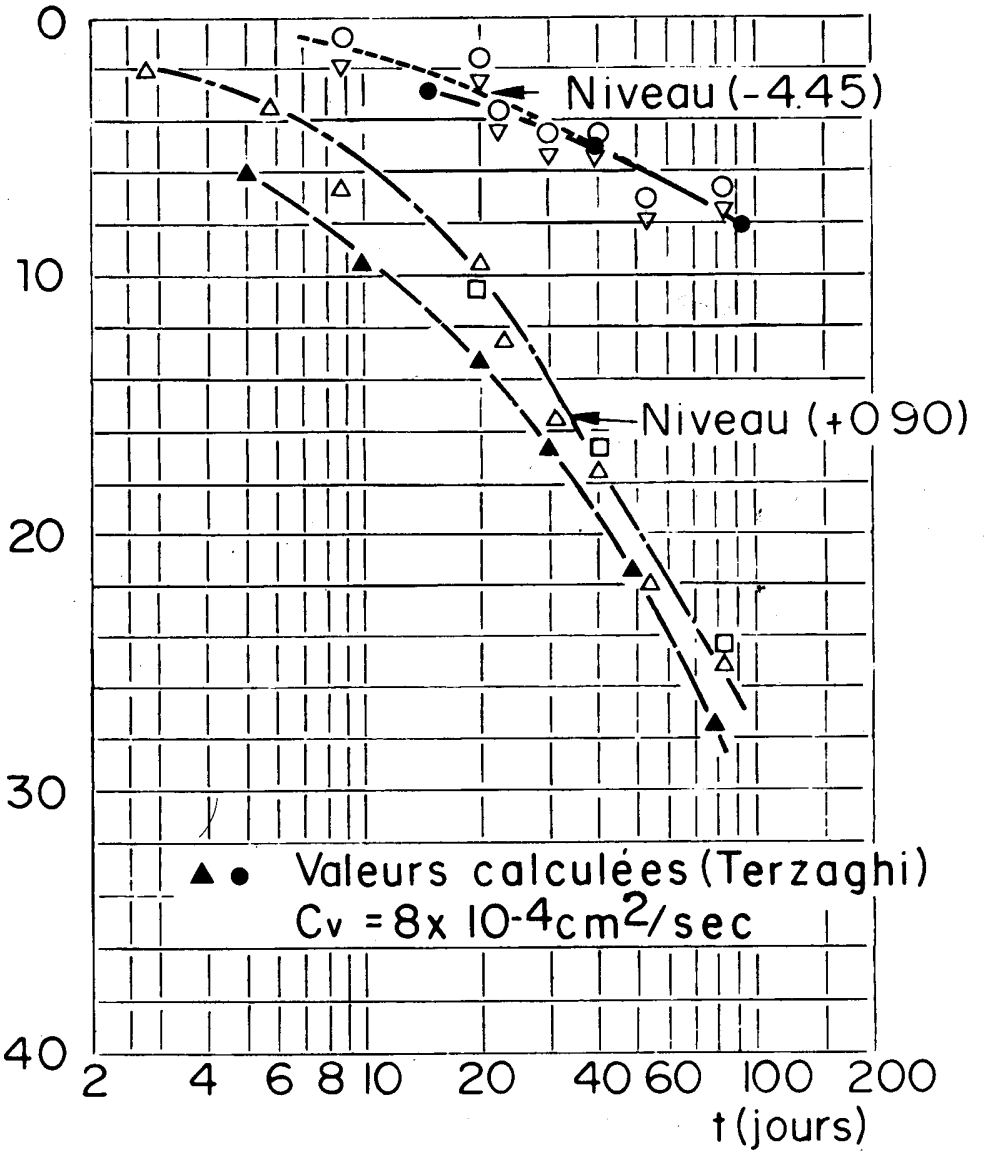

Fig. 9 Terrassements mesurés

Fig. 10 Pressions interstitielles mesurées

Surpression interstitielle $\mathrm{KPa}$

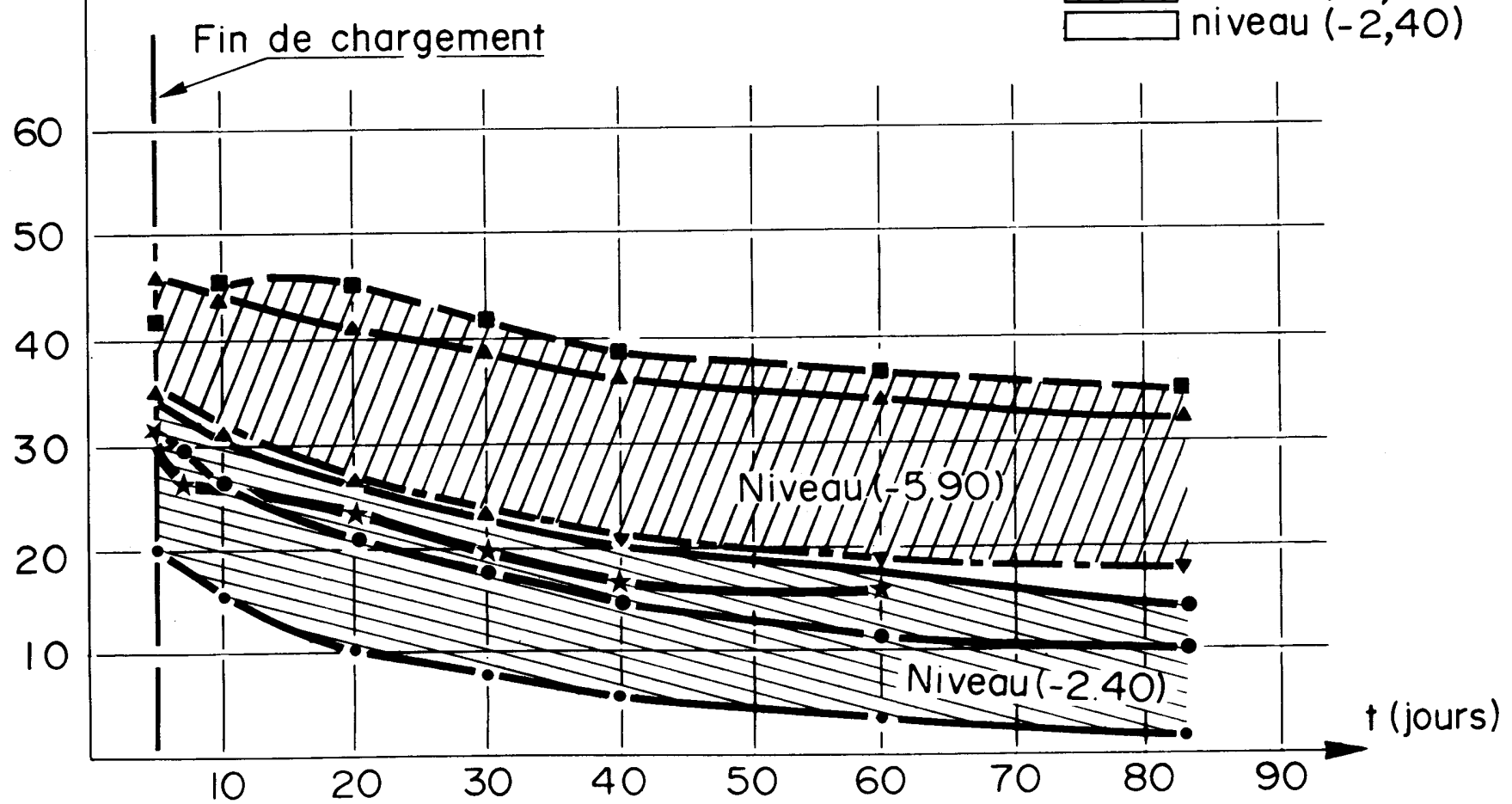

\section{Légende}

fuseaux des mesures

पIII] niveau $(-5,90)$

$\square$ niveau $(-2,40)$ 


\section{Tentative de conclusions}

Il est bien difficile de tirer des conclusions scientifiques trop précises dans le cas présent, en raison même des moyens et temps d'expérimentation limites disponibles. Néanmoins sur le plan pratique ces remblais d'essais ont permis de prendre les décisions concernant le projet des digues d'accès.

a) seul l'essai en vraie grandeur a permis de constater le comportement réel de la fondation des digues d'accès à créer, sous réserve que la fondation testée sous les deux remblais d'essais soit bien représentative de l'ensemble de la fondation. Ce qui en moyenne semble être le cas.

b) l'auscultation, limitée pour des raisons d'économie, aurait mérité d'être complétée par des cellules de pressions dans des sondages de pied (hypothèse de la redistribution des pressions interstitielles et du drainage latéral). Quelques clinomètres de pied auraient pu être prévus pour suivre le fluage. Des tassomètres du type téléniveau auraient certainement posé moins de problèmes lors de l'exécution et permis des mesures plus précises.

c) II semble bien que le facteur correcteur de Bjerrum soit exagérément défavorable, dans ce cas particulier, même si l'on tient compte des effets d'extrémités et peut être d'une certaine cohésion du remblai proprement dit, qui n'a pas été mesurée.

d) les tassements mesurés sont, comme on le constate souvent, supérieurs aux tassements calculés par la théorie simplifiée de Terzaghi (anisotropie de la fondation, remaniement difficile à éviter des prélèvements), encore serait-il nécessaire de compléter l'observation topographique, au-delà des 90 jours après la saison d'hivernage. e) au niveau des travaux à venir, les remblais seront construits en une seule phase avec une provision pour tassement de l'ordre de $1 \mathrm{~m}$ à $1,50 \mathrm{~m}$. L'auscultation topographique précise prévue en cours de travaux apportera des éléments complémentaires (sur le fluage en particulier) qui permettront de préciser la revanche à donner, en fin de construction pour tenir compte des tassements ultérieurs de la fondation.

\section{Références Bibliographiques}

[1] J. MANDEL et J. SALENCON. - Force portante d'un sol sur une assise rigide $7^{\circ}$ Congrès International de Mécanique des Sols Mexico - 1969 - Vol. 2 - Page 157.

[2] L. BJERRUM. - $8^{\circ}$ Congrès International de Mécanique des Sols Moscou 1973 - Tome 3 - Pages 111 à 152.

[3] Études des remblais sur sols compressibles. Recommandations des laboratoires des Ponts et Chaussées - Dunod Paris 1971.

[4] MM. BALIGH et AS AZZOUZ - ASCE - GT 11 - End effects on stability of cohesive slapes. - Novembre 1975.

[5] Remblais sur sols compressibles - Bulletin de liaison des laboratoires, des Ponts et Chaussées. - Spécial T - Mai 1973.

[6] AW. BISHOP et L. BJERRUM. - The relevance of the triaxial test to the solution of stability problems. - ASCE - University of Colorado - Juin 1960. 